\title{
ESTRATIFICAÇÃO VERTICAL EM ÁREA DE COLETA DE SEMENTES DA TERRA INDÍGENA PARAKANÃ, ESTADO DO PARÁ, BRASIL
}

Noemi Vianna Martins Leão ${ }^{\text {; }}$ José Francisco Pereira ${ }^{2}$; Sérgio Heitor Sousa Felipe ${ }^{3}$; Antônia Camila dos Santos Moraes ${ }^{4}$; Osvaldo Ryohei Kato ${ }^{5}$

${ }^{1}$ Pesquisadora da Embrapa Amazônia Oriental, Belém, Pará, Brasil. noemi.leao@embrapa.br

${ }^{2}$ Pesquisador da Embrapa Amapá, Macapá, Amapá, Brasil.

${ }^{3}$ Doutor em Botânica, Universidade Federal de Viçosa, Viçosa, Minas Gerais, Brasil.

${ }^{4}$ Engenheira Florestal pela Universidade Federal Rural da Amazônia, Belém, Pará,

Brasil.

${ }^{5}$ Pesquisador da Embrapa Amazônia Oriental, Belém, Pará, Brasil.

\section{Recebido em: 06/04/2019 - Aprovado em: 10/06/2019 - Publicado em: 30/06/2019} DOI: 10.18677/EnciBio_2019A63

\begin{abstract}
RESUMO
O estudo da estratificação vertical da floresta evidencia a dominância de determinadas espécies em relação ao comportamento ecológico. Adicionalmente, também pode auxiliar na definição dos métodos de escalada para a colheita de frutos e sementes na copa de árvores. O presente trabalho teve como objetivo analisar a estrutura vertical com base nos indivíduos que compõe as diferentes famílias botânicas na Área de Coleta de Sementes (ACS) da Terra Indígena Parakanã (T.I. Parakanã), estado do Pará. Foi determinada a altura de 10.383 indivíduos presentes na ACS da T.I. Parakanã pelo método de estimação visual. Posteriormente, foram definidas cinco classes de altura (classe superior 1; superior 2; médio 1; médio 2 e inferior) para distribuição dos indivíduos. Através da análise multivariada as famílias foram agrupadas por similaridade igual a $70 \%$. Os resultados evidenciaram que as famílias Anacardiaceae, Fabaceae, Lauraceae, Lecythidaceae e Moraceae compõem o dossel. As diferentes famílias botânicas formaram seis grupos considerando similaridade igual a $70 \%$. O grupo 1 e 2, com 29 famílias, constitui o estrato superior da floresta, no entanto, também apresentam indivíduos nas classes inferiores; os grupos 3 e 4, somando 14 famílias, constituem prioritariamente o dossel intermediário e o sub-bosque; o grupo 5 , equivalente a 7 famílias, localiza-se $100 \%$ no dossel intermediário, e, em relação ao grupo 6 , formado somente por 2 famílias, os indivíduos encontram-se no sub-bosque em sua totalidade. Os diferentes estratos observados na ACS da T.I. Parakanã evidencia a necessidade do uso de diferentes métodos de escalada para colheita de frutos e sementes.
\end{abstract}

PALAVRAS-CHAVE: estratos arbóreos, floresta ombrófila, manejo florestal, posição sociológica. 


\title{
VERTICAL STRATIFICATION IN AREA SEED COLLECTION FROM PARAKANÃ INDIGENOUS LAND, PARÁ STATE, BRAZIL
}

\begin{abstract}
The study of the vertical stratification of the forest evidences the dominance of certain species in relation to the ecological behavior. Additionally, it can also support in the definition of climbing methods for harvesting fruits and seeds in the tree canopy. The present work aimed to analyze the vertical structure based on the individuals that compose the different botanical families in the Seed Collection Area (SCA) belong Parakanã Indigenous Land (PIL), Pará state. The height of 10,383 individuals present at ACS of T.I. Parakanã was determined by the visual estimation method. Subsequently, five height classes (superior class 1, superior 2, middle 1, middle 2 and inferior) were defined for the individuals' distribution. By the multivariate analysis the families were grouped by similarity equal to $70 \%$. The results showed that the families Anacardiaceae, Fabaceae, Lauraceae, Lecythidaceae and Moraceae belong to the SCA canopy. The different botanical families formed six groups considering similarity equal to 70\%. Group 1 and 2, with 29 families, constitute the superior stratum of the forest, however, also have individuals in the inferior classes; groups 3 and 4, with 14 families, constitute the intermediate canopy and sub-forest; group 5, equivalent to 7 families, is located $100 \%$ in the intermediate canopy, and, in relation to group 6 , formed only by 2 families, the individuals are in the total sub-forest. The different strata observed in the SCA of PIL evidences the need to use different climbing methods to harvest fruits and seeds.
\end{abstract}

KEYWORDS: tree layers strata, ombrophilous forest, forest management, sociologic position.

\section{INTRODUÇÃO}

No Brasil, as Terras Indígenas (T.I.) estão divididas em 721 áreas, sendo 424 concentradas na Região Amazônica, correspondendo a 58,8\% em relação ao território nacional. O Estado do Pará possui 64 das 424 Terras Indígenas, com área equivalente a $26,7 \%$ do total de Terras Indígenas na região (FUNAI, 2018). Dentre as etnias indígenas presente na Amazônia, estado do Pará, têm-se o povo Parakanã.

A Terra Indígena Parakanã (T.I. Parakanã) está localizada no sudeste do Pará, situada a oeste do rio Tocantins, sendo considerada região integrante dos municípios de Novo Repartimento e Itupiranga. Atualmente, compreende 15 aldeias: Paranowaona (PNW), Paranatinga (PNT), Parano'a (PNO), Paranoawe (PNA), Itaygo'a (ITG), Inaxyganga (IXG), Maroxewara (MXW), Itapeyga (ITP), Paranoita (PNI), Itaoenawa (ITW), O'ayga (OYG), Paranoema (PNE), Itaygara (ITY), Xataopawa (XTP) e Xaraira (XRI) (PROGRAMA PARAKANÃ, 2019).

Em 1986, foi criado o Programa Parakanã, na qual está inserido o Subprograma de Produção que possibilitou o desenvolvido do projeto "Coleta de Frutos e Sementes de Espécies Florestais". Este projeto visa à geração de renda por meio da coleta de frutos e sementes de diferentes espécies florestais nativas anualmente (PROGRAMA PARAKANÃ, 2019). Essa atividade é fundamental para a manutenção e preservação da floresta devido à exploração de frutos e sementes como recursos florestais não madeireiros (LEÃO et al., 2015).

A vegetação da T.I. Parakanã é classificada como Floresta Ombrófila Densa Submontana, que possui alta riqueza de espécies com árvores de grande porte, 
alcançando 50 metros ou mais. Apresenta sub-bosque rico em regeneração natural e a presença de palmeiras de pequeno porte, além de lianas lenhosas e alta densidade de epífitas, o que é um fator de diferenciação desta tipologia (IBGE, 2012).

A estrutura florestal está fundamentalmente ligada a processos funcionais que controlam o provisionamento de serviços ecossistêmicos em paisagens florestais (THOM ; KEETON, 2019). Nesse contexto, a análise da estrutura vertical em florestas pode evidenciar, dependendo, da região fitoecológica, estádio de sucessão e estado de conservação, a presença de sub-bosque, como o estrato inferior, estrato médio e estrato superior, ou não apresentar estratificação evidente (ARAÚJO et al., 2016; GUIMARÃES et al., 2016; FREITAS ; MAGALHÃES, 2016; MIGUEL et al., 2016). Além disso, o conhecimento dos estratos presentes em áreas de florestas nativas fornece informações que podem auxiliar diretamente no plano de colheita de frutos e sementes florestais.

Estudos de estratificação vertical desenvolvido em floresta de várzea na Área de Proteção Ambiental llha do Combu, Pará (BRAGA et al., 2015), bem como avaliação vertical do dossel em uma área da Mata Atlântica, Parque Estadual da Serra do Mar, São Paulo (CONTO et al., 2015), evidenciaram que a estratificação vertical influencia a riqueza, diversidade, crescimento e produção de biomassa, pois há competição natural entre as espécies por luz.

Segundo Ali (2009), ao avaliar diversos estudos sobre estrutura e funcionamento da floresta, observou que pesquisas sobre estratificação vertical em florestas geram informações que contribuem diretamente para o manejo florestal devido revelar se há aumento da biomassa acima do solo ou no armazenamento de carbono através das interações positivas planta-planta sob 0 efeito de complementariedade do habitat.

Em razão da importância do assunto é necessário que mais estudos sejam realizados nas florestas primárias e secundárias da região amazônica para auxiliar no manejo sustentável e na conservação das áreas cobertas por florestas. Deste modo, o presente trabalho teve como objetivo analisar a estrutura vertical com base nos indivíduos que compõe as diferentes famílias botânicas na Área de Coleta de Sementes da Terra Indígena Parakanã, estado do Pará.

\section{MATERIAL E MÉTODOS}

A área experimental está localizada na aldeia Paranowaona (PNW), norte da T.I. Parakanã, que se situa entre os Municípios de Novo Repartimento e Itupiranga, estado do Pará (Figura 1). O clima da região segundo a classificação de Köppen é do tipo Am, com temperatura média anual variando entre 24 e $26^{\circ} \mathrm{C}$. A precipitação pluviométrica anual é superior a $2.000 \mathrm{~mm}$, distribuída com regularidade e com presença de período seco entre os meses de junho a setembro (SUDAM, 1984). 


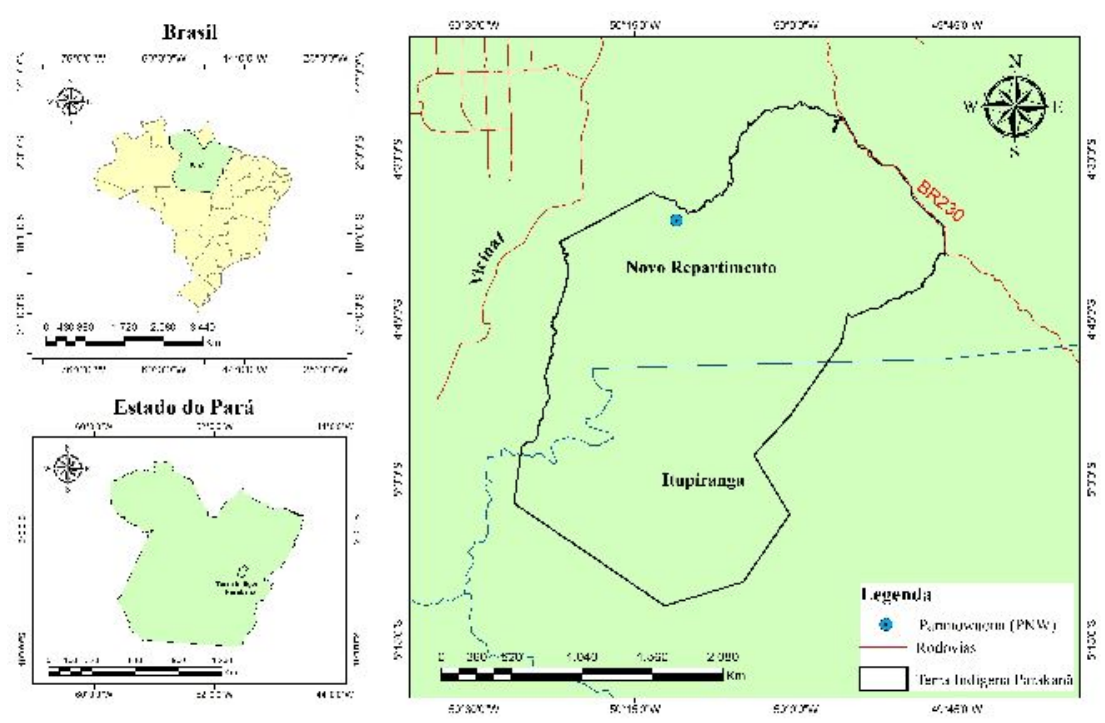

FIGURA 1. Mapa da localização da aldeia Paranowaona na Terra Indígena Parakanã, Novo Repartimento, estado do Pará. Fonte: Leão et al. (2018).

Através da interpretação de imagens de satélites foram realizados estudos fitofisionômicos e classificada a cobertura florestal existente na T.I. Parakanã próximo à Aldeia Paranowaona. Após esta análise, juntamente com os índios, foi selecionado o local mais apropriado para a implantação da Área de Coleta de Sementes, considerando-se a acessibilidade, a distância da aldeia mais próxima e a presença de espécies de valor econômico e ecológico como o mogno (Swietenia macrophylla King), pau-preto (Cenostigma tocantinum Ducke) e castanha-do-pará (Bertholletia excelsa Bonpl.).

A metodologia de demarcação da Área de Coleta de Sementes e seleção das espécies foi de acordo com Leão et al. (2005). A demarcação e o piqueteamento foi feita com a instalação de 25 parcelas de 200 m x 200 m (4 hectares), subdivididas em 16 subparcelas de $50 \mathrm{~m}$ x $50 \mathrm{~m}$. Foram identificadas as espécies botânicas e demarcadas todas as árvores com diâmetro a altura do peito (DAP) maior ou igual a $25 \mathrm{~cm}$. Adicionalmente, também foi mensurada a altura do fuste e a altura total desses indivíduos, exceto para as palmeiras. A medição da altura foi realizada pelo método de estimação visual com o auxílio de régua graduada de 10 metros, em que a régua era apoiada à árvore para criar uma referência para o observador.

O material botânico foi identificado por técnicos do Museu Paraense Emílio Goeldi - MPEG, sendo confeccionadas exsicatas para confirmação taxonômica por meio de comparações ou uso de chave de identificação botânica. Inicialmente, foi adotado o sistema de classificação de CRONQUIST (1988), e a sinonímia botânica foi confirmada no Herbário do MPEG. A classificação foi atualizada adotando-se o sistema do Jardim Botânico do Rio de Janeiro, Flora do Brasil 2020, na qual se baseia nas circunscrições propostas por APG III (REFLORA, 2014).

A estrutura vertical da Área de Coleta de Sementes considerou 10.383 indivíduos no inventário com DAP maior ou igual a $25 \mathrm{~cm}$. Posteriormente, foram definidas cinco classes de altura (classe superior 1; superior 2; médio 1; médio 2 e inferior) para análise por meio do Software Mata Nativa Stat. Aplicou-se a análise multivariada de agrupamento pelo método de Dendrograma com Ligação Completa e a Distância Euclidiana, considerando similaridade de $70 \%$ com base na distribuição relativa dos indivíduos. Essa técnica objetiva dividir em grupos, visando 
aproximar dados semelhantes e obter elevada heterogeneidade entre os grupos formados. A análise multivariada foi realizada por através do Software Minitab versão 14.0.

\section{RESULTADOS E DISCUSSÃO}

Os resultados da ACS da T.I. Parakanã evidenciou que os 10.383 indivíduos, com diâmetro a altura do peito (DAP) maior ou igual a $25 \mathrm{~cm}$, encontram-se distribuídos em 52 famílias botânicas. A análise dos dados dos 10.383 indivíduos apresentou a seguinte distribuição absoluta: i) extrato superior 1, com altura entre 56 e $69 \mathrm{~m}$, tem um total de 05 indivíduos; ii) superior 2, com altura entre 43 e $56 \mathrm{~m}$, possui 190 indivíduos; iii) médio 1, com altura entre 30 e $43 \mathrm{~m}$, equivalente a 1.641 indivíduos; iv) médio 2, com altura entre 17 e 30 m, apresenta 5.802 indivíduos; e, v) inferior, com altura entre 04 e $17 \mathrm{~m}$, com o total de 2.745 indivíduos (Tabela 1).

A estrutura vertical da floresta é importante indicador de sustentabilidade do manejo florestal, pois reflete a quantidade de energia existente na floresta (SOUSA NETO et al., 2018). No presente estudo observou-se que ACS T.I. Parakanã apresenta estratos bem definidos (variando de sub-bosque a dossel florestal), com a presença de indivíduos da família Fabaceae e Meliaceae compondo todos os estratos. A distribuição dos indivíduos nos diferentes estratos da floresta está diretamente ligada à dinâmica de populações das espécies, sendo influenciada por fatores intrínsecos (produção e tipos de frutos e sementes, ciclo de vida, exigência de luz) e extrínsecos (ataque de insetos e patógenos, predação de frutos e sementes) (DE PAULA et al., 2004); assim, indivíduos dessas famílias podem ser considerados como melhores adaptados ao dossel florestal (CALLEGARO et al., 2012).

No presente estudo, a maioria dos indivíduos encontra-se no estrato médio, representando $71,7 \%$ do total. Esse resultado corrobora com Dionisio et al. (2016) e Condé e Tonini (2013) que encontraram em cerca de $70 \%$ dos indivíduos ocorrendo na classe de altura média ao analisarem áreas florestais no estado de Roraima. Esses mesmos autores ressaltam que poucos indivíduos alcançam o dossel da floresta. Essa resposta observada no presente estudo pode estar relacionada com a competição entre os indivíduos, tanto em combinações intra como interespecíficas, por disponibilidade de água, nutrientes e, principalmente, luz (PIRES-O'BRIEN ; O'BRIEN, 1995). Nesse sentido, para que muitas espécies alcancem o dossel superior é necessário o surgimento de pequenas clareiras.

TABELA 1- Número absoluto de indivíduos distribuídos em cinco classes de alturas de acordo com cada família botânica encontrada na Área de Coleta de Sementes da Terra Indígena Parakanã, Novo Repartimento, estado do Pará.

\begin{tabular}{clcccccc}
\hline Códig & \multirow{2}{*}{ Família } & \multicolumn{7}{c}{ Classes (metros) } & \multirow{2}{*}{ Total } \\
\cline { 3 - 7 } 0 & & $4 \mid---17$ & $17 \mid---30$ & $30 \mid--43$ & $43 \mid---56$ & $56 \mid---69$ & \\
\hline 1 & Anacardiaceae & 17 & 75 & 92 & 12 & 0 & 196 \\
2 & Annonaceae & 21 & 69 & 40 & 2 & 0 & 132 \\
3 & Apocynaceae & 25 & 81 & 36 & 2 & 0 & 144 \\
4 & Araliaceae & 1 & 2 & 3 & 0 & 0 & 6 \\
5 & Bignoniaceae & 1 & 29 & 39 & 4 & 0 & 73 \\
6 & Boraginaceae & 8 & 8 & 0 & 0 & 0 & 16 \\
7 & Burseraceae & 1424 & 2722 & 88 & 3 & 0 & 4237 \\
8 & Cardiopteridaceae & 1 & 1 & 0 & 0 & 0 & 2
\end{tabular}




\begin{tabular}{|c|c|c|c|c|c|c|c|}
\hline 9 & Caricaceae & 14 & 8 & 0 & 0 & 0 & 22 \\
\hline 10 & Caryocaraceae & 0 & 1 & 1 & 0 & 0 & 2 \\
\hline 11 & Celastraceae & 1 & 1 & 0 & 0 & 0 & 2 \\
\hline 12 & Chrysobalanaceae & 39 & 109 & 20 & 0 & 0 & 168 \\
\hline 13 & Clusiaceae & 7 & 23 & 4 & 1 & 0 & 35 \\
\hline 14 & Combretaceae & 1 & 12 & 30 & 1 & 0 & 44 \\
\hline 15 & Connaraceae & 0 & 3 & 0 & 0 & 0 & 3 \\
\hline 16 & Dichapetalaceae & 0 & 1 & 0 & 0 & 0 & 1 \\
\hline 17 & Ebenaceae & 1 & 5 & 1 & 0 & 0 & 7 \\
\hline 18 & Elaeocarpaceae & 4 & 2 & 1 & 0 & 0 & 7 \\
\hline 19 & Euphorbiaceae & 23 & 53 & 24 & 1 & 0 & 101 \\
\hline 20 & Fabaceae & 715 & 1120 & 615 & 84 & 4 & 2538 \\
\hline 21 & Goupiaceae & 0 & 3 & 0 & 0 & 0 & 3 \\
\hline 22 & Humiriaceae & 1 & 8 & 2 & 0 & 0 & 11 \\
\hline 23 & Icacinaceae & 1 & 0 & 0 & 0 & 0 & 1 \\
\hline 24 & Indeterminada & 6 & 9 & 3 & 0 & 0 & 18 \\
\hline 25 & Lamiaceae & 1 & 0 & 0 & 0 & 0 & 1 \\
\hline 26 & Lauraceae & 39 & 174 & 128 & 11 & 0 & 352 \\
\hline 27 & Lecythidaceae & 51 & 79 & 96 & 34 & 0 & 260 \\
\hline 28 & Linaceae & 0 & 2 & 0 & 0 & 0 & 2 \\
\hline 29 & Malpighiaceae & 5 & 5 & 0 & 0 & 0 & 10 \\
\hline 30 & Malvaceae & 72 & 217 & 56 & 14 & 0 & 359 \\
\hline 31 & Melastomataceae & 0 & 3 & 0 & 0 & 0 & 3 \\
\hline 32 & Meliaceae & 88 & 169 & 20 & 3 & 1 & 281 \\
\hline 33 & Moraceae & 46 & 219 & 103 & 14 & 0 & 382 \\
\hline 34 & Myristicaceae & 3 & 49 & 42 & 0 & 0 & 94 \\
\hline 35 & Myrtaceae & 5 & 11 & 1 & 0 & 0 & 17 \\
\hline 36 & Nictaginaceae & 37 & 13 & 1 & 0 & 0 & 51 \\
\hline 37 & Ochnaceae & 0 & 1 & 0 & 0 & 0 & 1 \\
\hline 38 & Olacaceae & 3 & 6 & 2 & 0 & 0 & 11 \\
\hline 39 & Opiliaceae & 1 & 1 & 0 & 0 & 0 & 2 \\
\hline 40 & Peraceae & 1 & 9 & 5 & 0 & 0 & 15 \\
\hline 41 & Polygonaceae & 1 & 4 & 0 & 0 & 0 & 5 \\
\hline 42 & Rhamnaceae & 28 & 103 & 11 & 0 & 0 & 142 \\
\hline 43 & Rosaceae & 0 & 1 & 0 & 0 & 0 & 1 \\
\hline 44 & Rubiaceae & 3 & 13 & 4 & 0 & 0 & 20 \\
\hline 45 & Rutaceae & 3 & 35 & 12 & 0 & 0 & 50 \\
\hline 46 & Salicaceae & 12 & 90 & 28 & 1 & 0 & 131 \\
\hline 47 & Sapindaceae & 4 & 12 & 5 & 0 & 0 & 21 \\
\hline 48 & Sapotaceae & 12 & 76 & 46 & 1 & 0 & 135 \\
\hline 49 & Simaroubaceae & 5 & 28 & 37 & 1 & 0 & 71 \\
\hline 50 & Ulmaceae & 7 & 15 & 1 & 0 & 0 & 23 \\
\hline 51 & Urticaceae & 6 & 111 & 32 & 0 & 0 & 149 \\
\hline \multirow[t]{2}{*}{52} & Vochysiaceae & 1 & 11 & 12 & 1 & 0 & 25 \\
\hline & Total & 2745 & 5802 & 1641 & 190 & 5 & 10383 \\
\hline
\end{tabular}

*Não houve êxito na identificação/confirmação botânica desses indivíduos. 
As famílias Anacardiaceae, Fabaceae, Lauraceae, Lecythidaceae e Moraceae apresentaram indivíduos com altura superior a $43 \mathrm{~m}$. Essas famílias contribuem com diferentes espécies para a formação do dossel da floresta da ACS da T.I. Parakanã. Esses resultados facilitam a definição de ações de manejo para sua conservação, como a seleção adequada do método a ser utilizado para a colheita de frutos $\mathrm{e}$ sementes.

Na família Fabaceae, com maior contribuição de indivíduos na classe superior 1 e 2, foram detectados elevado número de indivíduos de Alexa grandiflora Ducke. Além disso, também foram observadas as presenças de Albizia elegans (Ducke) L.Rico, Apuleia leiocarpa (Vogel) J.F.Macbr., Balizia elegans (Ducke) Barneby \& J.W.Grimes, Cenostigma tocantinum Ducke, Hymenaea courbaril L., Hymenaea intermedia Ducke, Hymenaea reticulata Ducke, Hymenolobium excelsum Ducke, Parkia multijuga Benth., Parkia pendula (Willd.) Benth. ex Walp., Parkia velutina Benoist, Pseudopiptadenia psilostachya (DC.) G.P.Lewis \& M.P.Lima, Pterocarpus officinalis Jacq., Schizolobium amazonicum Huber ex Ducke, Taralea oppositifolia Aubl. e Vatairea sericea (Ducke) Ducke com indivíduos maiores ou iguais a $43 \mathrm{~m}$.

Estudo envolvendo a análise da estrutura e uso potencial de espécies arbóreas em floresta manejada, em Santarém, PA, encontrou a espécie Alexa grandiflora entre as dez mais abundantes e com maior área basal (CORRÊA et al., 2015). Ressalta-se que essa espécie é endêmica do Brasil com ocorrência no Amazonas, Amapá e Pará (REFLORA, 2017). Segundo Salomão et al. (1995), Alexa grandiflora apresenta porte grande em relação ao diâmetro e alcança o dossel da floresta, corroborando com o resultado encontrado neste estudo.

Em relação à família Lecythidaceae, o comportamento foi semelhante ao da família Fabaceae, com a presença de uma espécie destacando-se pela maior abundância. Nessa família foram encontrados elevado número de indivíduos de Bertholletia excelsa. Essa espécie possui exemplares com alturas acima do dossel, chamadas de árvores plus ou gigantes da floresta (SILVA ; LEÃO, 2006). Também apresentou a ocorrência de Couratari oblongifolia Ducke \& Kunth e Lecythis zabucajo Aubl. A família Lecythidaceae contempla os tauaris (gênero Couratari) que têm por característica principal a altura de suas árvores e o tamanho das copas que ao longe são avistadas (LEÂO et al., 2005).

Para família Anacardiaceae e Lauraceae foi observada somente a presença de indivíduos de duas espécies com alturas maiores ou iguais a $43 \mathrm{~m}$. A família Anacardiaceae com presença de indivíduos de Astronium gracile Engl. e Astronium lecointei Ducke e a Lauraceae com indivíduos de Mezilaurus itauba (Meisn.) Taub. ex Mez e Ocotea rhynchophylla (Meisn.) Mez. Em relação à família Moraceae, foram verificadas a presença de Bagassa guianensis Aubl., Brosimum acutifolium Huber, Clarisia racemosa Ruiz \& Pav., Ficus amazonica (Miq.) Miq. e Ficus gomelleira Kunth.

A presença de somente determinadas espécies no dossel da floresta está relacionada às maiores exigências por luminosidade e competitividade entre as espécies (LAMBRECHT et al., 2016). Adicionalmente, o extrato inferior é formado, muitas vezes, por espécies que são exclusivas desse estrato, porém, são fundamentais para a composição da estrutura florestal (MENDES et al., 2012).

Através da análise multivariada e considerando a similaridade igual a $70 \%$, observou-se a existência de seis grupos (Figura 2), o que pode ser indicativo de floresta multiestratificada. 


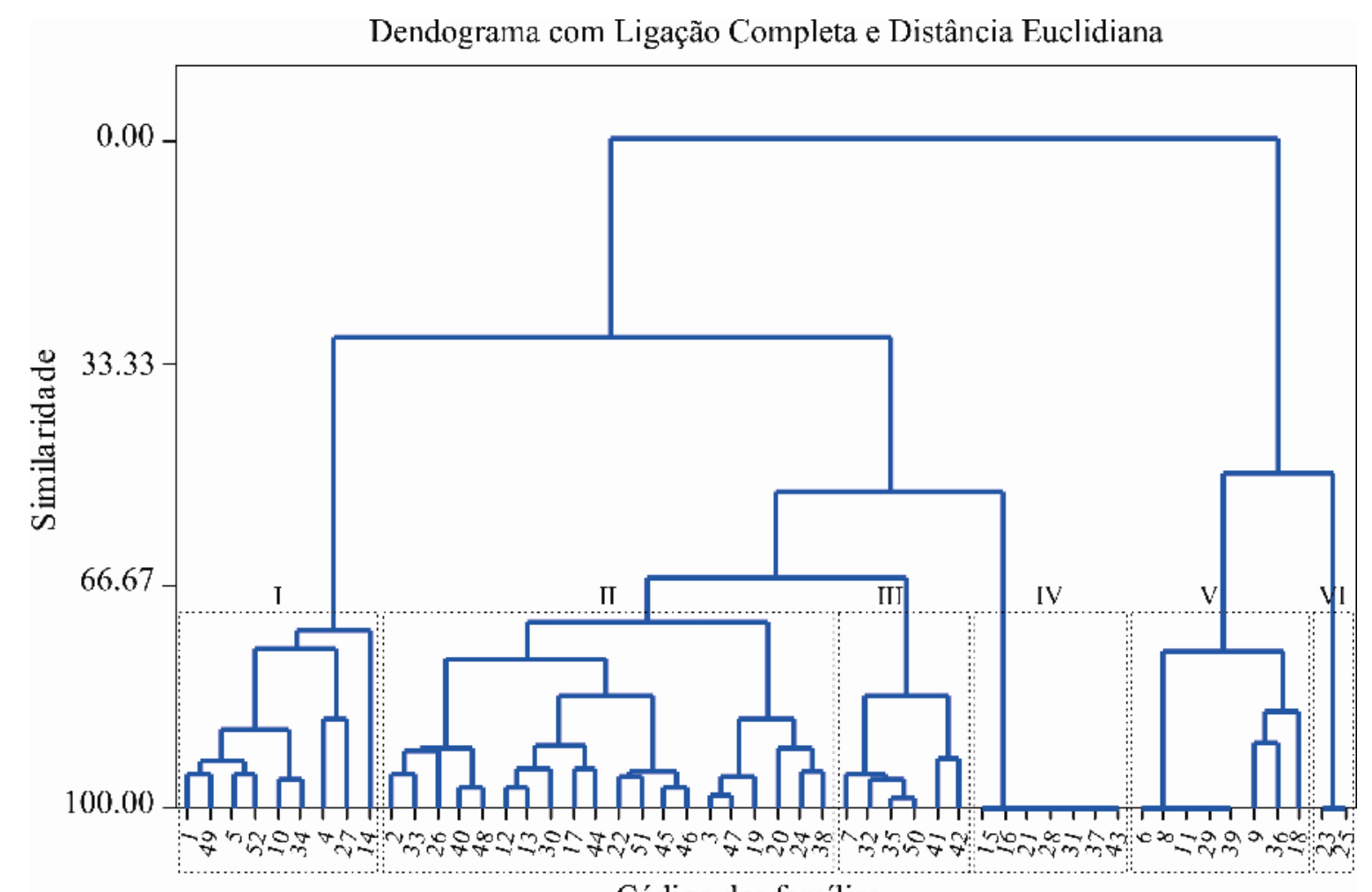

Código das famílias

FIGURA 2- Dendograma evidenciando o agrupamento das famílias na Área de Coleta de Sementes da Terra Indígena Parakanã, em Novo Repartimento, estado do Pará.

A distribuição da altura dos 10.383 indivíduos foi ajustada a uma curva exponencial negativa. A maioria das árvores apresenta altura até $30 \mathrm{~m}(82 \%$ dos indivíduos), compondo o sub-bosque florestal, enquanto o dossel superior é formado por $2 \%$ dos indivíduos (Figura 1). Ressalta-se que esse dossel é formado principalmente por indivíduos da espécie Alexa grandiflora, o que evidencia ser uma espécie de caráter dominante na ACS.

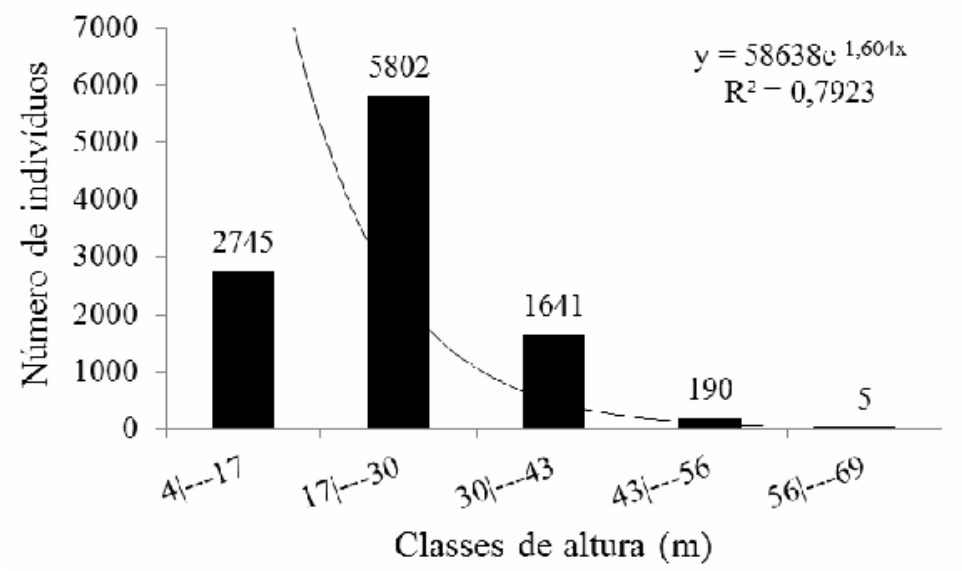

FIGURA 3- Distribuição de frequências observadas e ajustadas em classes de alturas de 10.383 indivíduos da Área de Coleta de Sementes da Terra Indígena Parakanã, Novo Repartimento, estado do Pará. 
Para o melhor conhecimento da distribuição dos indivíduos de cada família nos estratos da ACS da Terra Indígena Parakanã verificou-se também a distribuição dos mesmos nas cinco classes de altura a partir da formação dos seis grupos de familias com similaridade de $70 \%$ gerado pelo dendograma de ligação completa e distância euclidiana. O grupo 1 foi formado por 9 famílias (Anacardiaceae, Bignoniaceae, Caryocaraceae, Myristicaceae, Simaroubaceae, Vochysiaceae, Araliaceae, Lecythidaceae e Combretaceae). Nesse grupo 52,5\% dos indivíduos apresentaram altura superior a $30 \mathrm{~m}$, destacando-se a classe de estrato médio 2 (30 a $43 \mathrm{~m}$ de altura), com a presença de $45,7 \%$ dos indivíduos (Figura $4 \mathrm{~A}$ ).

O grupo 2 foi formado por 20 famílias (Annonaceae, Apocynaceae, Chrysobalanaceae, Clusiaceae, Ebenaceae, Euphorbiaceae, Fabaceae, Humiriaceae, Indeterminada, Lauraceae, Malvaceae, Moraceae, Olacaceae, Peraceae, Rubiaceae, Rutaceae, Salicaceae, Sapindaceae, Sapotaceae e Urticaceae). Nesse grupo os indivíduos distribuíram-se em todas as classes de altura. Contudo, $21,7 \%$ dos indivíduos estão na classe inferior (árvores com altura até $30 \mathrm{~m}$ ) e $51 \%$ na classe média 1 (árvores com altura variando entre 17 e $30 \mathrm{~m}$ ), e, somente $27,2 \%$ dos indivíduos estão com altura acima de $30 \mathrm{~m}$ (Figura 4B).

O grupo 3 foi formado por 6 famílias (Burseraceae, Meliaceae, Myrtaceae, Polygonaceae, Rhamnaceae e Ulmaceae). Nesse grupo 33,0; 64,3 e 2,6\% dos indivíduos estão na classe inferior, médio 1 e 2, respectivamente (Figura 4C). O grupo 4 foi formado por 8 famílias (Boraginaceae, Cardiopteridaceae, Caricaceae, Celastraceae, Elaeocarpaceae, Malpighiaceae, Nictaginaceae e Opiliaceae). Nesse grupo 63,4 e 34,8\% dos indivíduos estão na classe inferior (altura variando entre 4 e $17 \mathrm{~m}$ ) e médio 1 (altura variando entre $17 \mathrm{e} 30 \mathrm{~m}$ ), respectivamente. Soma-se a isso, o fato dessa distribuição, nessas duas classes, representar $98,2 \%$ do total de indivíduos (Figura 4D).

O grupo 5 foi formado por 7 famílias (Connaraceae, Dichapetalaceae, Goupiaceae, Linaceae, Melastomataceae, Ochnaceae e Rosaceae). Nesse grupo $100 \%$ dos indivíduos encontram-se na classe médio 1 (altura variando entre 17 e 30 $\mathrm{m}$ ) (Figura 4E). O grupo 6 foi formado somente por 2 famílias (Icacinaceae e Lamiaceae). Nesse grupo 100\% dos indivíduos encontra-se na classe inferior (altura variando entre 4 e $17 \mathrm{~m}$ ) (Figura 4F). Porém, é importante ressaltar que para cada família desse grupo foi encontrado somente um indivíduo durante o inventário florístico. 

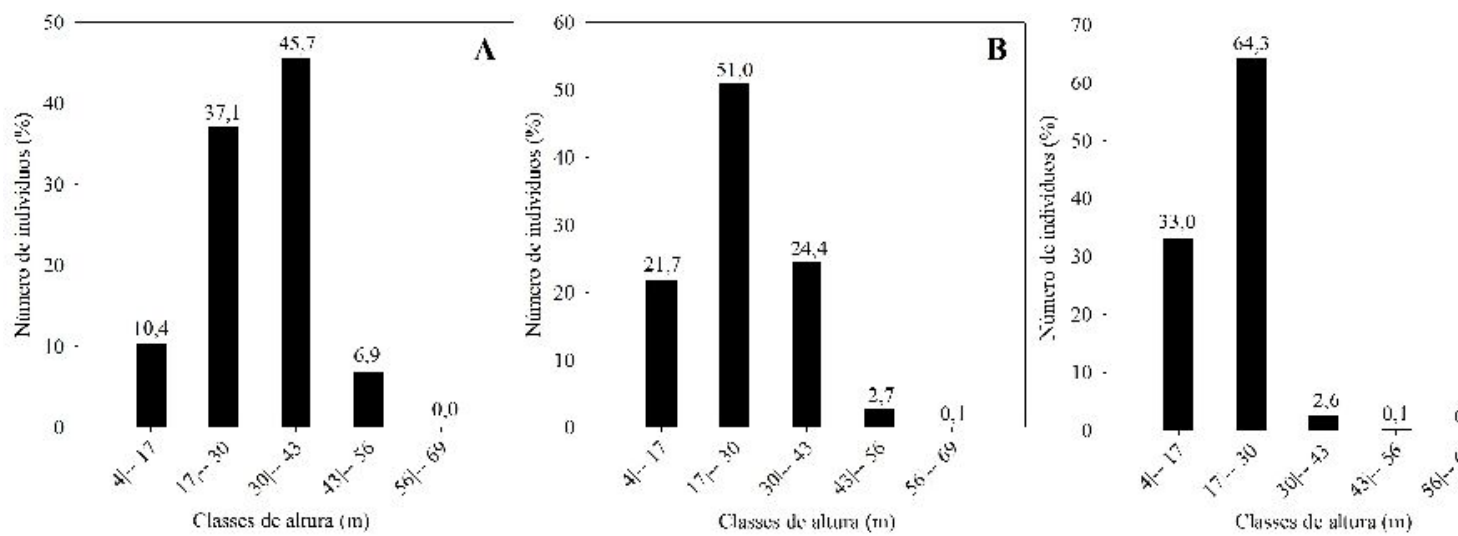

C
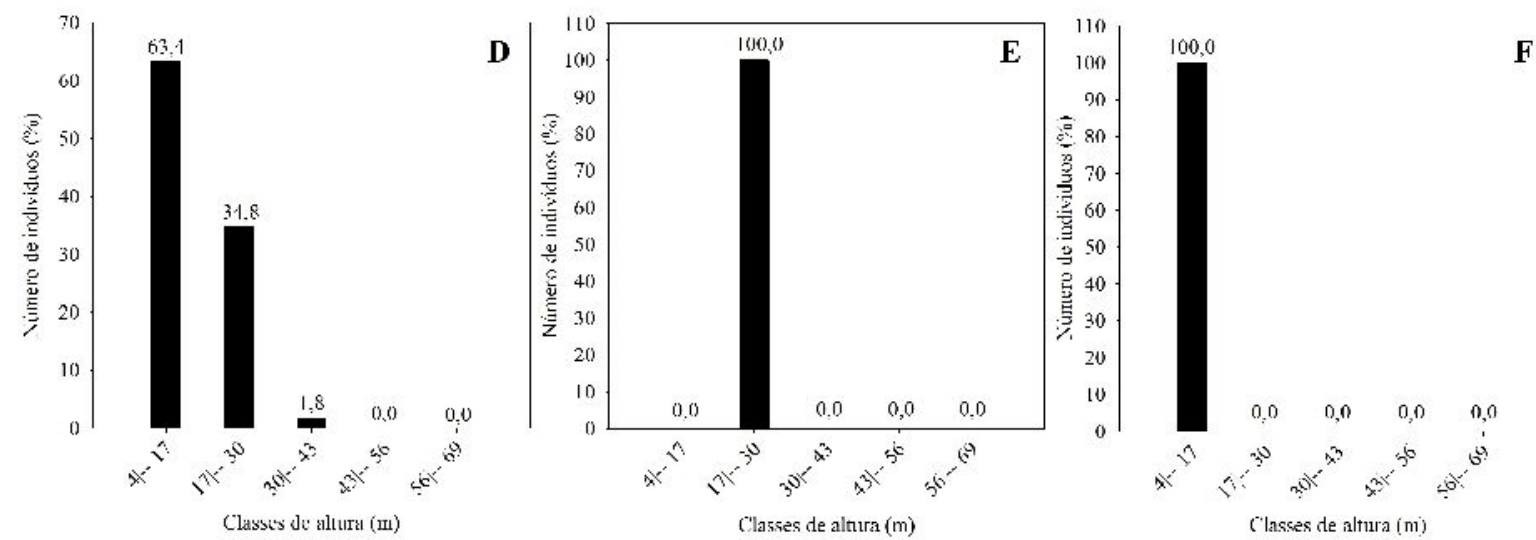

FIGURA 4- Porcentagem de indivíduos distribuídos nas cinco classes de altura na Área de Coleta de Sementes da Terra Indígena Parakanã, em Novo Repartimento, estado do Pará. Grupo 1 (A); Grupo 2 (B); Grupo 3 (C); Grupo 4 (D); Grupo 5 (E) e Grupo 6 (F).

Os resultados desta pesquisa evidenciam que a ACS da Terra Indígena Parakanã apresenta indivíduos de diferentes alturas, como muito baixa (entre 4 e 17 $\mathrm{m}$ ), baixa (entre 17 e $30 \mathrm{~m}$ ), média (entre 31 e $43 \mathrm{~m}$ ), alta (entre 44 e $56 \mathrm{~m}$ ) e muito alta (entre 57 e $69 \mathrm{~m}$ ). A distribuição desses indivíduos nos estratos reflete a dinâmica florestal, que está fortemente ligada ao ciclo e hábito de vida dos mesmos, pois determinadas famílias restringem-se às determinadas alturas, não apresentam ampla distribuição nos estratos. Além disso, algumas espécies podem não alcançar o estrato superior de uma floresta devido não se reproduzirem ou regenerarem no local e outras são típicas do estrato inferior e médio (VIEIRA et al., 2015).

\section{CONCLUSÕES}

A Área de Coleta de Sementes da T.I. Parakanã apresenta seis grupos baseado na distribuição relativa dos indivíduos nas cinco classes de alturas (superior 1 e superior 2, médio 1, médio 2 e inferior). 0 grupo 1 e 2, totalizando 29 famílias, constituem o estrato superior da floresta estudada, no entanto, apresentam indivíduos em todas as classes. Os grupos 3 e 4, somando 14 famílias, constituem prioritariamente o dossel intermediário e o sub-bosque. O grupo 5, com 7 famílias, localiza-se $100 \%$ no dossel intermediário. Em relação ao grupo 6, que reúne somente 2 famílias os indivíduos encontram-se no sub-bosque em sua totalidade.

Recomenda-se o uso de diferentes métodos de escalada para colheita de frutos e sementes (p.ex. método de esporas com cinto de segurança, método de 
alpinismo/rapel, método do blocante ao tronco e método de escada de alumínio) na Área de Coleta de Sementes da T.I. Parakanã.

\section{AGRADECIMENTOS}

À Fundação Amazônia de Amparo a Estudos e Pesquisas e ao Programa Parakanã/ Eletronorte pelo apoio técnico e suporte.

\section{REFERÊNCIAS}

$\mathrm{ALI}, \mathrm{A}$. Forest stand structure and functioning: Current knowledge and future challenges. Ecological Indicators, v. 98, p. 665-677, 2019. https://doi.org/10.1016/j.ecolind.2018.11.017.

ARAÚJO, A. C. B.; ARAUJO, H. J. B.; CALLEGARO, R. M.; ANDRZEJEWSKI, C.; LONGHI, S. J. Estrutura de dois componentes arbóreos de floresta subtropical ripária na campanha gaúcha, Sant'ana do Livramento, RS. Revista Floresta, v. 46, n. 4, p. 481-490, 2016. http://dx.doi.org/10.5380/rf.v46i4.42590.

BRAGA, E. O.; FRANÇA, J. A.; PANTOJA, M. V.; JARDIM, M. A. G. Florística, estrutura fitossociológica e formas de vida do estrato inferior em uma floresta de várzea Amazônica. Biota Amazônia, v. 5, n. 3, p. 59-65, 2015. http://dx.doi.org/10.18561/2179-5746/biotaamazonia.v5n3p59-65.

CALLEGARO, R. M.; LONGHI, S. J.; ARAUJO, A. C. B.; KANIESKI, M. R.; FLOSS, P. A.; GRACIOLI, C. R. Estrutura do componente arbóreo de uma floresta estacional decidual ripária em Jaguari, RS. Ciência Rural, v. 42, n. 2, p. 305-311, 2012. http://dx.doi.org/10.1590/S0103-84782012000200019.

CALLEGARO, R. M.; ANDRZEJEWSKI, C.; JONAS LONGHI, S.; VILLANOVA LONGHI, R.; JOB BIALI, L. Composição das categorias sucessionais na estrutura horizontal, vertical e diamétrica de uma Floresta Ombrófila Mista Montana. Revista Brasileira de Ciências Agrárias, v. 11, n. 4, p. 350-358, 2016. http://dx.doi.org/10.5039/agraria.v11i4a5406.

CONDÉ, T. M.; TONINI, H. Fitossociologia de uma Floresta Ombrófila Densa na Amazônia Setentrional, Roraima, Brasil. Acta Amazonica, v. 43, n. 3, p. 247-260, 2013. http://dx.doi.org/10.1590/S0044-59672013000300002.

CONTO, T.; GÖRGENS, E. B.; SILVA, A. G. P.; LARANJA, D. C. F.; RODRIGUEZ, L. C. E. Caracterização do perfil vertical do dossel de um trecho de Mata Atlântica através de escaneamento laser aerotransportado. Scientia Forestalis, v. 43, n. 108, p. 873-884, 2015. http://dx.doi.org/10.18671/scifor.v43n108.12.

CORRÊA, V. V.; GAMA, J. R. V.; RIBEIRO, R. B. D. S.; ALVES, A. F.; VIEIRA, D. D. S.; XIMENES, L. C. Estrutura e uso potencial de espécies arbóreas em floresta manejada, PA Moju, Santarém-Pará. Revista Cerne, v. 21, n. 2, p. 293-300, 2015. http://dx.doi.org/10.1590/01047760201521021488.

CRONQUIST, A. The evolution and classification of flowering plants. New York: The New York Botanical Garden, 1988. 
DE PAULA, A. D.; SILVA, A. F.; MARCO JÚNIOR, P.; SANTOS, F. A. M., SOUZA, A. L. Sucessão ecológica da vegetação arbórea em uma Floresta Estacional Semidecidual, Viçosa, MG, Brasil. Acta Botanica Brasilica, v. 18, n. 3, p. 407-423, 2004. http://dx.doi.org/10.1590/S0102-33062004000300002.

DIONISIO, L. F. S.; BONFIM FILHO, O. S.; SOUZA CRIVELLI, B. R.; GOMES, J. P.; OLIVEIRA, M. H. S.; CARVALHO, J. O. P. Importância fitossociológica de um fragmento de floresta ombrófila densa no estado de Roraima, Brasil. Revista Agro@mbiente On-Line, v. 10, n. 3, p. 243-252, 2016. http://dx.doi.org/10.18227/1982-8470ragro.v10i3.3381.

FREITAS, W. K. D.; MAGALHÃES, L. M. S. Vertical structure of the arboreal component under regeneration phase of a deciduous forest fragment, Santa Catarina state, Brazil. Revista Árvore, v. 40, n. 5, p. 781-791, 2016. http://dx.doi.org/10.1590/0100-67622016000500002.

FUNAI. Índios no Brasil. 2018. Disponível em: http://www.funai.gov.br/. Acesso em: 03 Mar. 2019.

GUIMARÃES, J. R. S.; CARIM, M. D. J. V.; TOSTES, L. D. C. L.; COSTA NETO, S. V. Floristic diversity of secondary forest in the eastern amazon, state of Amapá.

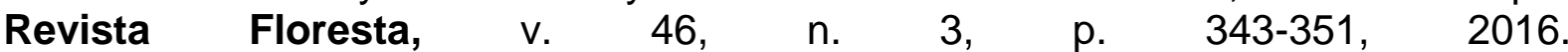
http://dx.doi.org/10.5380/rf.v46i3.40161.

IBGE - Instituto Brasileiro de Geografia e Estatística. Manual Técnico da Vegetação Brasileira: Sistema Fitogeográfico; Inventário das formações florestais e campestres; técnica e manejo de coleções botânicas; procedimentos para mapeamento. Rio de Janeiro, 2012. Disponível

em: https://biblioteca.ibge.gov.br/visualizacao/livros/liv63011.pdf. Acesso em: 03 Mar. 2019.

LAMBRECHT, F. R.; DALLABRIDA, J. P.; TRAUTENMÜLLER, J. W.; CARLI, L.; BURGIN, M. R. B.; FORTES, F. D. O. Florística e estrutura em área de Floresta Estacional Decidual na região do Alto Uruguai, RS. Revista Brasileira de Engenharia de Biossistemas, v. 10, n. 2, p. 198-209, 2016. http://dx.doi.org/10.18011/bioeng2016v10n2p198-209.

LEÃO, N. V. M.; FELIPE, S. H. S.; SILVA, C. E.; MORAES, A. C. S.; SHIMIZU, E. S. C.; GALLO, R.; FREITAS, A. D. D.; KATO, O. R. Morphometric diversity between fruits and seeds of mahogany trees (Swietenia macrophylla King.) from Parakanã Indigenous Land, Pará State, Brazil. Australian Journal of Crop Science, v. 12, p. 435-443, 2018.

LEÃO, N. V. M.; OHASHI, S. T.; FELIPE, S. H. S. Situação atual da pesquisa e produção de sementes de espécies florestais nativas na Amazônia Oriental. In: PIÑA-RODRIGUES, F. C. M.; FIGLIOLIA, M. B.; SILVA, A. (Org.). Sementes Florestais Tropicais: da ecologia à produção. 01 ed., Londrina-PR: ABRATES, 2015, v. 01, p. 381-395. 
LEÃO, N. V. M.; OHASHI, S. T.; VIEIRA, I. C. G.; GHILARDI JR., R. Ilha de Germoplasma de Tucuruí: uma reserva da biodiversidade para o futuro. $01 \mathrm{ed}$. Brasília: Eletronorte, 2005. v. 01. p. 232.

MENDES, F. S.; JARDIM, F. C. S.; CARVALHO, J. O. P.; LIMA, T. T. S.; SOUZA, D. V. Dinâmica da composição florística do sub-bosque em floresta tropical manejada, no município de Moju, estado do Pará, Brasil. Revista de Ciências Agrárias, v. 55, n. 2, p. 117-123, 2012. http://dx.doi.org/10.4322/rca.2012.050.

MIGUEL, E. P.; REZENDE, A. V.; LEAL, F. A.; PEREIRA, R. S.; MELO, R. R. D. Floristic-structural characterization and successional group of tree species in the Cerrado Biome of Tocantins state, Brazil. Revista Caatinga, v. 29, n. 2, p. 393-404, 2016. http://dx.doi.org/10.1590/1983-21252016v29n216rc.

PIRES-O'BRIEN, M.J.; O'BRIEN, C.M. Ecologia e modelamento de florestas tropicais. Belém: FCAP, 1995. 400p.

PROGRAMA PARAKANÃ. Programas Indígenas Eletrobras/ Eletronorte. Disponível em:

http://www.eletronorte.gov.br/opencms/opencms/pilares/meioAmbiente/programasIn digenas/parakana/. Acesso em: 03 Mar. 2019.

REFLORA. Fabaceae in Flora do Brasil 2020 em construção. Jardim Botânico do Rio de Janeiro. Disponível em: http:/floradobrasil.jbrj.gov.br/reflora/floradobrasi/FB78995. Acesso em: 20 Jan. 2017.

REFLORA. Lista de espécies da Flora do Brasil. Jardim Botânico do Rio de Janeiro. Disponível em: http://floradobrasil.jbrj.gov.br/. Acesso em: 15 Dez. 2014.

SALOMÃO, R. P.; ROSA, N. A.; NEPSTAD, D. C.; BAKK, A. Estrutura diamétrica e breve caracterização ecológica-econômica de 108 espécies arbóreas da floresta amazônica brasileira. Revista Interciência, v. 20, n. 1, p. 20-9, 1995. Disponível em: http://www.interciencia.org/v20_01/art03/.

SILVA, S.; LEÃO, N. V. M. Árvores da Amazônia. São Paulo, SP: Empresa das Artes, 2006. p. 243.

SOUSA NETO, E. N.; DE PAULA, A.; TAGLIAFERRE, C.; BARRETO-GARCIA, P. B.; LONGUE JÚNIOR, D. Performance assessment of methodologies for vertical stratification in native forests. Ciência Florestal, v. 28, n. 4, p. 1583-1591, 2018. http://dx.doi.org/10.5902/1980509835106.

SOUZA, D. R.; SOUZA, A. L. Estratificação vertical em floresta ombrófila densa de terra firme não explorada, Amazônia Oriental. Revista Árvore, v. 28, n. 5, p. 691698, 2004. http://dx.doi.org/10.1590/S0100-67622004000500008.

SUDAM. Atlas climatológico da Amazônia Brasileira. Belém: SUDAM. Projeto de Hidrologia e Climatologia da Amazônia (PHCA), 1984. 
THOM, D.; KEETON, W. S. Stand structure drives disparities in carbon storage in northern hardwood-conifer forests. Forest Ecology and Management, v. 442, p. 1020, 2019. https://doi.org/10.1016/j.foreco.2019.03.053.

VIEIRA, D. S.; GAMA, J. R. V.; OLIVEIRA, M. L. R.; SILVA RIBEIRO, R. B. Análise estrutural e uso múltiplo de espécies arbóreas em florestas manejadas no médio vale do rio Curuá-Una, Pará. Revista Floresta, v. 45, n. 3, p. 465-476, 2015. http://dx.doi.org/10.5380/rf.v45i3.35584 